\title{
Assessing molluscs functional diversity within different coastal habitats of Mediterranean marine protected areas
}

\author{
Luigia Donnarumma*, Roberto Sandulli, Luca Appolloni, Giovanni Fulvio Russo
}
Department of Science and Technology, Laboratory of Marine Ecology, University of Naples "Parthenope", Centro Direzionale - Isola C4, 80143, Naples, Italy. National Interuniversity Consortium for Marine Sciences (CoNISMa), Rome, Italy. e-mail: luigia.donnarumma@uniparthenope.it

Received: 15 May 2018 / Accepted: 12 July 2018

\begin{abstract}
Molluscs assemblages inhabiting subtidal habitats (sandy bottoms, photophilic and sciaphilic hard bottoms, Posidonia oceanica seagrass beds) represent a valuable part of coastal biodiversity and were studied in four Italian Marine Protected Areas. Quantitative samplings were carried out in late spring - summer of 2015. A total of 776 individuals were identified, belonging to the classes of Polyplacophora (5 species, 24 individuals), Gastropoda (62 sp., 240 ind.), Bivalvia (51 sp., 488 ind.), and Scaphopoda (3 sp., 24 ind.). Multivariate analyses revealed significant inter-habitat differences in the composition of mollusc assemblages, especially between soft bottom and all the other habitats. In all MPAs, the highest species richness and feeding guilds occurred in the photophilic hard bottom, where, in contrast, a lower number of individuals was found. On the contrary, in the soft bottom the highest number of individuals and the lower species richness and feeding guilds were found. Although the results pointed out some intrahabitat differences among MPAs, the molluscan assemblages showed a valuable degree of taxonomic and trophic similarity, especially in vegetated habitats. These results are of primary importance for ecosystem functioning and management as the considered habitats are the most relevant of the coastal zones.
\end{abstract}

Keywords: Mollusc assemblages, trophic groups, subtidal habitats, Mediterranean Sea

\section{Introduction}

Molluscs are among the more diverse groups of the marine benthic communities, occupying a wide range of habitats and ecological niches and largely contributing to local biodiversity (Hemminga and Duarte, 2000; Zuschin et al., 2001). In the Mediterranean Sea more than 2,000 molluscs species have been identified and almost 53,000 have been described worldwide, especially in costal zones (Bouchet, 2006; Coll et al., 2010). This world-wide distribution is mainly due to the ability of the molluscs to adapt to different forcing factors and food sources, with appropriate and diversified functional morphologies (Russo, 1989;
Chemello et al., 1997). Groups of species exploiting the same class of environmental resources in a similar way are defined by Root (1967) as 'guilds', that is the name of medieval association of craftsmen.

Beyond taxonomic composition of marine assemblages, trophic guilds can be studied to better understand the functional diversity, as they express the main energy pathway of ecosystems and, indirectly, the main physical forcing factors of environment (Díaz and Cabido, 2001; Arruda et al., 2003; Vassallo et al., 2017). Habitat heterogeneity greatly influences the associated mollusc communities through a variety of energy pathways, increasing species diversity which, in turn, generates a more structured 
community with a greater trophic complexity and feeding mode, despite the dominance of single or few guilds at a defined time (Lastra et al., 1991; Zuschin et al., 2001; Tews et al., 2004). For instance, a higher variety of grazer snails is related to habitat with high primary production, as Posidonia oceanica beds and rocky photophilic habitats (Milazzo et al., 2000; Pitacco et al., 2014). Predator species are dominant in tropical reefs characterized by a high diversity of reef building animals (Paine, 1969), while, suspension and deposit feeders mainly occur in soft bottom surrounded and characterized by high amount of particulate organic matter (Heip et al., 1995; Newell and Clavier, 1997).

Among marine systems, coastal zones are the richest in species and habitats and, therefore, are also the most threatened. As a consequence, conservation policy and management strategies for these habitats have been more and more implemented in the last decades (Cicin-Sain and Belfiore, 2005; Meiner, 2010; Katsanevakis et al., 2011). Along the Italian shores, Marine Protected Areas (MPAs) have been instituted by the law 394/91 and, together with the Habitat Directive (92/43/EEC) on European species and habitats of priority importance for conservation, they represent a useful tool to protect marine biodiversity and to implement sustainable practises of coastal zone management (Browman et al., 2004; Halpern et al., 2010; Rassweiler et al., 2012). Several studies demonstrate the effectiveness of MPAs for the conservation of marine biodiversity (Appolloni et al., 2017, 2018; Ferrigno et al., 2017, 2018), the increase of ecosystem services and the production of ecological and economic benefits that are exported in the surrounding coastal areas (i.e., Guidetti et al., 2008; Fenberg et al., 2012; Franzese et al., 2008; 2015; 2017; Picone et al., 2017). In this framework, it is very important to assess how the assemblages of those systematic groups, as molluscs, with high levels of biodiversity are structured. This is particularly true in MPAs to evaluate habitats quality and assess management actions.

Molluscan assemblages might be effectively used in environmental quality assessment (Gladstone, 2002), being generally distributed from shallow to deep marine water, in different types of habitats such as seagrass beds (Russo et al., 1984; Russo and Patti, 2005; Gacia et al., 2009; Donnarumma et al., 2016; 2018a), photophilic algae communities (Chemello and Russo, 1997; Chemello and Milazzo, 2002), coralligenous concretions (Ballesteros et al., 2006; Casellato and Stefanon, 2008), caves (Cattaneo-Vietti and Russo, 1987), soft bottoms (Russo et al., 1985; Urra et al., 2011), and rocky shores (Richards, 1983; Terlizzi et al., 2003; Antoniadou and Chintiroglou, 2005; Donnarumma et al., 2018b). Thus, the present investigation aims at assessing the structure and function (through the use of feeding guilds) of molluscan assemblages inhabiting different habitat types and comparing them among different Italian
MPAs. Taking into account the positive effect of MPAs protection on the biodiversity, we test the following hypotheses: (i) among habitats, there is a higher trophic and species diversity in the most spatially complex ones, (ii) among MPAs, there are no significant differences in the composition of mollusc assemblages within the same type of habitat.

\section{Materials and methods}

\subsection{Study areas and sampling design}

The study was carried out in four different Marine Protected Areas along a latitudinal gradient (Fig. 1). Three of them are located in Tyrrhenian Sea, [from north to south: Isole di Ventotene and Santo Stefano MPA (IVS) $40^{\circ} 47^{\prime} 08.59^{\prime \prime} \mathrm{N} 13^{\circ} 26^{\prime} 12.22^{\prime \prime} \mathrm{E}$, Santa Maria di Castellabate MPA (SMC) $40^{\circ} 14^{\prime} 46.35^{\prime \prime} \mathrm{N} 14^{\circ} 53^{\prime} 44.95^{\prime \prime} \mathrm{E}$, Costa degli Infreschi e della Masseta MPA (CIM) $40^{\circ} 00^{\prime} 05.46^{\prime \prime} \mathrm{N}$ $\left.15^{\circ} 26^{\prime} 21.04^{\prime \prime} \mathrm{E}\right]$, while the southernmost site is located in Ionian Sea [Capo Rizzuto MPA (CR) 38 53'29.66" N, $\left.17^{\circ} 5^{\prime} 55.42^{\prime \prime} \mathrm{E}\right]$. Within each MPA the following habitats were investigated: soft bottom (SB) mainly characterized by fine sandy-muddy sediment, Posidonia oceanica seagrass bed (HP), photophilic hard bottom covered by algal turf (PHB) and sciaphilic hard bottom covered by bioconstructions (SHB, coralligenous habitat).

Both SB and HP habitats were sampled at a depth range of $10-20 \mathrm{~m}$, PHB habitat at a depth of about $10 \mathrm{~m}$, and SHB habitat at a depth range of $28-33 \mathrm{~m}$. Samplings were performed by SCUBA divers during late spring - summer 2015. At each site (IVS, SMC, CIM, and $\mathrm{CR}$ ), three random replicates were collected by means of an air-lift pump (Bianchi et al., 2004) equipped with a $0.5 \mathrm{~mm}$ mesh bag.

On the hard bottoms (SHB and PHB) the sampling technique described by Chemello and Russo (1997) was adopted. This technique is based on the use of a $40 \times 40$ $\mathrm{cm}$ frame and is carried out in three steps: (I) sucking by air-lift pump the vagile fauna; (II) scraping by a spatula the sessile flora and fauna; (III) sucking again by air-lift pump to collect cryptic species of the substrate crevices. In the Posidonia beds (HP), vagile fauna was sucked by air-lift pump both on the leaf stratum and on the sand among the rhizomes (Russo et al., 1986), in a 40x40 cm frame. In the soft bottoms (SB), macrofauna was sucked by air-lift pump in a 50x50 cm frame, reaching a depth in the sediment of $20 \mathrm{~cm}$. Samples were fixed in $4 \%$ formaldehyde/seawater solution and successively macrobenthos was sorted in laboratory and molluscs were selected and identified at species level using a stereomicroscope. Mollusc nomenclature follows the World Register of Marine Species database (WoRMS; Editorial Board, 2018). 


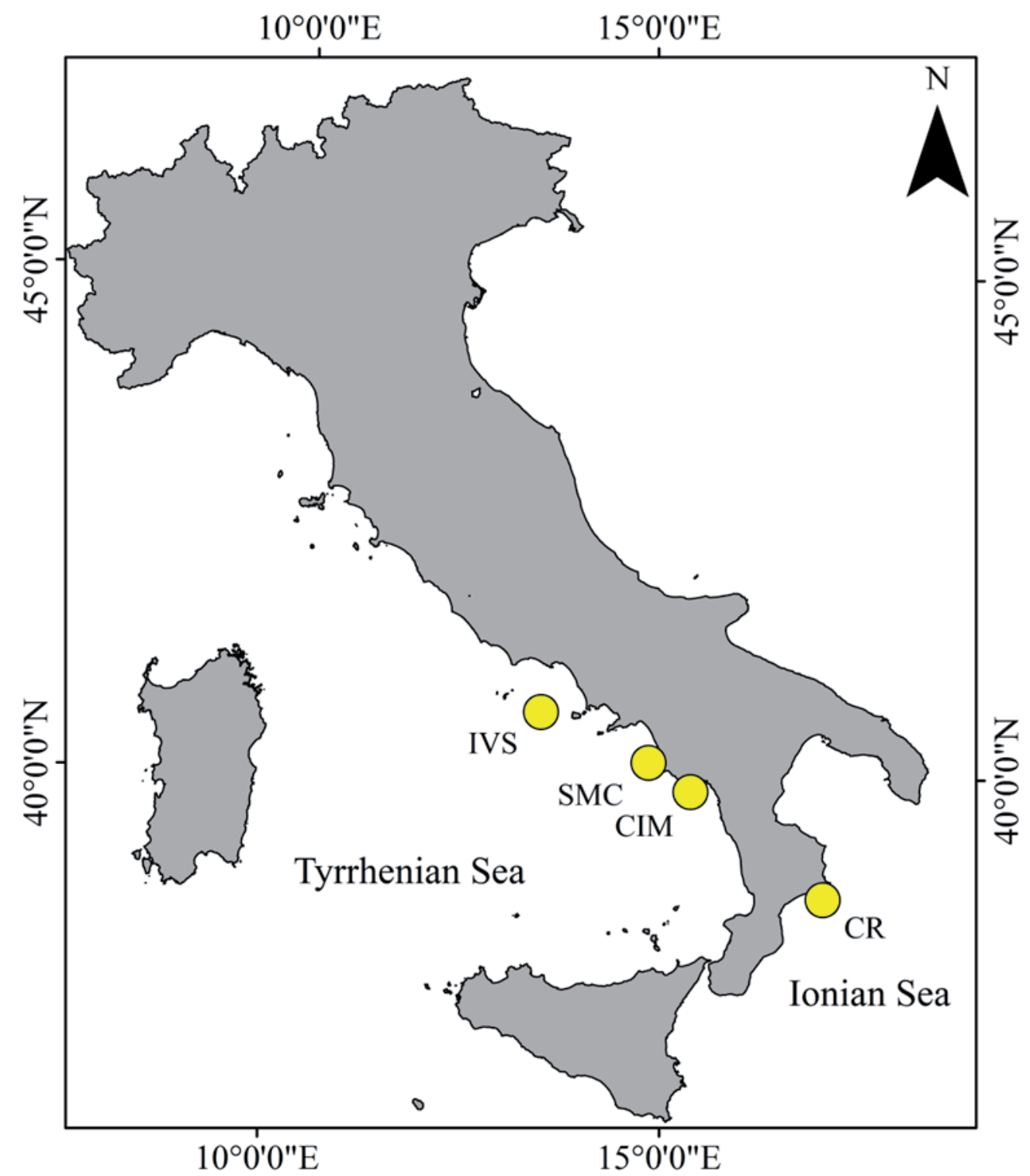

Fig. 1. Map of study area with the location of the four Marine Protected Areas investigated (IVS - Isole di Ventotene and Santo Stefano; SMC - Santa Maria di Castellabate; CIM - Costa degli Infreschi e della Masseta; CR - Capo Rizzuto)

\subsection{Data analysis}

Mollusc assemblages were analysed using sinecological indices such as total abundance of individuals $(\mathrm{N})$ per $\mathrm{m}^{-2}$, species richness (SR), the Pielou's Evenness (J) and the Shannon-Weaver species diversity ( $\left.\mathrm{H}^{\prime}\right)$. The quantitative and qualitative dominances (\%DI and \%DQ) and frequency $(\% \mathrm{~F})$ were also calculated. To each mollusc species a feeding guild was assigned, according to Purchon (1977), Fretter and Graham (1994) and Rueda et al. (2009). The following categories were considered: Micro- and Macro-Grazers (MG), feeding on both diatoms and/or algae; Deposit Feeders (DF), feeding on the organic particles of sediment; Suspension Feeders (SF), feeding on the organic particles suspended in the water; Predators (P), feeding on vagile and sessile animals; Scavengers (SC), feeding on dead animals; Ectoparasites (E), feeding on much larger animals.

The functional (trophic) diversity of the molluscan assemblages at each habitat was analysed through the Index of Trophic Diversity (ITD) (Help et al., 1998), which ranges from 0 to 1 (high and low trophic diversity, respectively), and is calculated according to the following equation:

$$
\operatorname{ITD}=\sum \mathrm{q}_{\mathrm{i}}^{2}
$$

where $q_{i}$ is the relative contribution of the number of individuals of each trophic group (i) to the total number of 
individuals. According to the modified version applied to molluscs (Antit et al., 2016), ITD was calculated as 1-ITD to have the highest trophic diversity with the greatest weight.

Differences in the structure of mollusc assemblages both in Habitats and Sites (two fixed and orthogonal factors) were evaluated through a permutational analysis of variance (PERMANOVA, Anderson, 2001a). 4999 permutations of residuals under a reduced model were applied (Anderson, 2001b) and a pairwise was used in order to evaluate differences between pairs of habitats and sites. Data were log transformed according to Clarke and Warwick (2001) to reduce the effect of the dominant species in the samples. Multivariate patterns were shown through a constrained ordination plot after Canonical Analysis of Principal Coordinates (CAP, Anderson and Willis, 2003). Similarity percentage (SIMPER) was performed to identify those species that better contributed to the similarity among the same habitats of the four sites. All multivariate analyses were performed using the PRIMER-PERMANOVA+v.6 software package (Clarke and Gorley, 2006; Anderson et al., 2008).

\section{Results}

\subsection{Composition of mollusc assemblages}

Overall, 121 species of molluscs, belonging to the classes of Polyplacophora (5 species; 24 individuals), Gastropoda
(62 sp.; 240 ind.), Bivalvia (51 sp.; 488 ind.), and Scaphopoda ( 3 sp.; 24 ind.) were identified in the four MPAs (Table 1).

In each habitat, a characteristic taxonomic group was dominant (Fig. 2). In the SB, bivalves were dominant both in species richness (DQ 63.8\%, 23 sp.) and abundance (DI $85 \%, 302$ individuals) followed by gastropods (DQ 27.7\%, 10 sp.; DI 8.1\%, 29 ind.) and scaphopods (DQ 8.3\%, 3 sp.; DI $6.7 \%, 24$ ind.). The most abundant and frequent species were the bivalves Lucinella divaricata and Moerella donacina (DI 43.9\%; F 83.3\% and DI 13\%; F 91.6\%, respectively), followed by the gastropod Tritia pellucida (DI $2.2 \%$; F 33.3\%) and the scaphopod Fustiaria rubescens (DI 5.6\%; F 25\%).

In PHB, gastropods were the dominant taxon both in species richness (DQ 62.2\%, $28 \mathrm{sp}$.) and abundance (DI $62.2 \%, 112$ ind.), followed by bivalves (DQ 28.8\%, $13 \mathrm{sp}$; DI 30\%, 54 ind.) and polyplacophorans (DQ 8.8\%, 4 sp.; DI $7.7 \%, 14$ ind.). The highest densities and frequencies were recorded by the gastropod species Bittium latreillii and Alvania subcrenulata (DI 11.6\%; 50\% and F 10.5\%; $25 \%$, respectively), followed by the bivalve Striarca lactea (DI 10\%; F 58.3\%) and the polyplacophoran Acanthochitona fascicularis (DI 2.7\%; F 33.3\%).

In SHB, bivalves were dominant in species richness (DQ 47.3\%, 18 sp.) and abundance (DI 59\%, 62 ind.), followed by gastropods (DQ 47.3\%, 18 sp.; DI 32.3\%, 34 ind.), while Polyplacophorans were poorly represented (DQ 5.2\%, 2 sp.; DI 8.5\%, 9 ind.). The most abundant and frequent species was the bivalve S. lactea (DI $28.5 \%$;
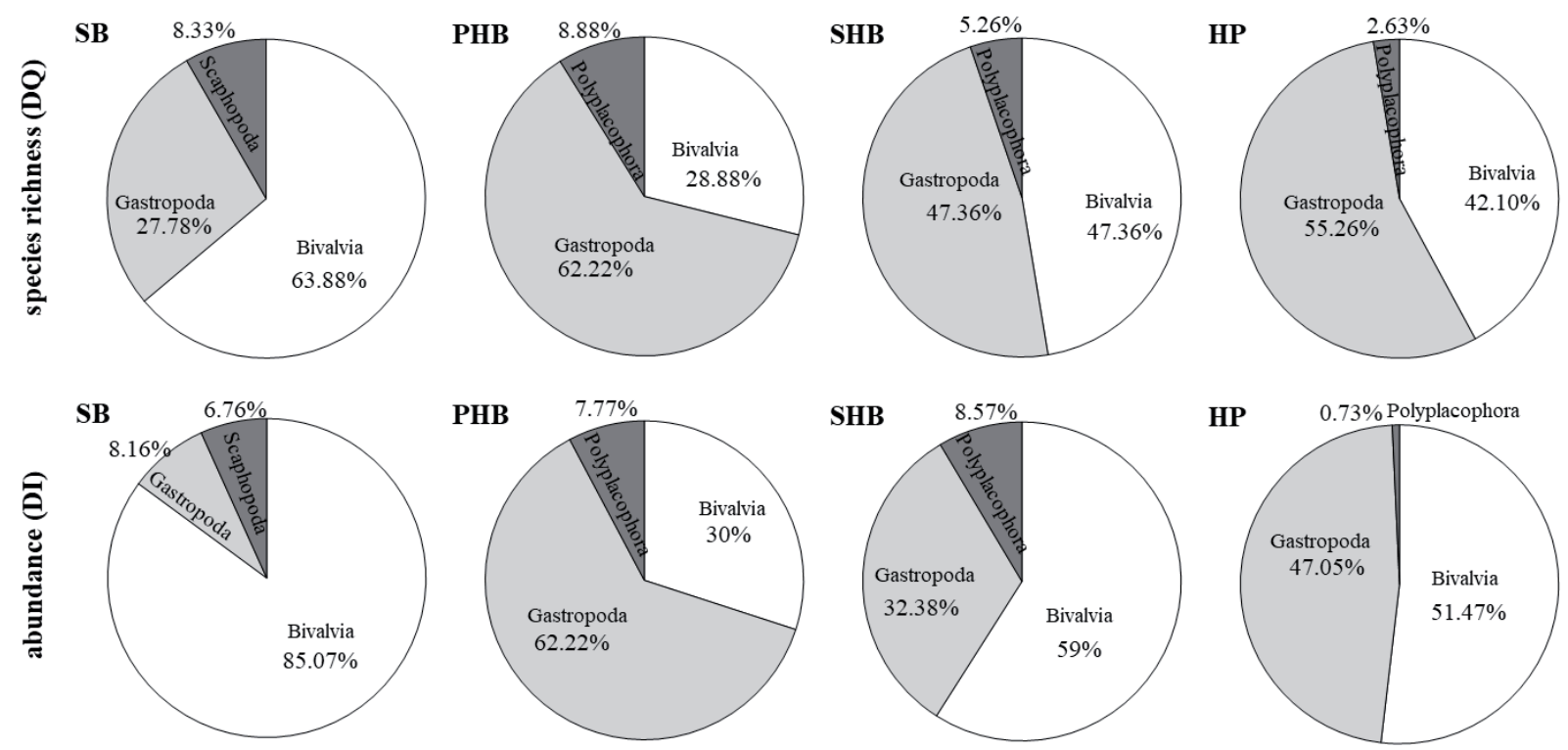

Fig. 2. Total contribution to qualitative (Species richness - \%DQ) and quantitative (Abundance - \%DI) dominances for each habitat (SB - soft bottom; PHB - photophilic hard bottom; SHB - sciaphilic hard bottom; HP - P. oceanica bed) 
Table 1. Taxonomic list of mollusc species with their feeding guilds (FG: MG Micro- and Macro-grazers, DF Detritus feeders, E Ectoparasites, SF Suspension feeders, P Predators, SC Scavengers), abundance (N), dominance (D\%) and frequency (F\%) for each habitat (SB - soft bottom; PHB - photophilic hard bottom; SHB - sciaphilic hard bottom; HP - P. oceanica bed)

\begin{tabular}{|c|c|c|c|c|c|c|c|c|c|c|c|c|c|}
\hline \multirow[b]{2}{*}{ FG } & \multirow[b]{2}{*}{ species } & \multicolumn{3}{|c|}{ SB } & \multicolumn{3}{|c|}{ PHB } & \multicolumn{3}{|c|}{ SHB } & \multicolumn{3}{|c|}{ HP } \\
\hline & & $\mathrm{N}$ & $\mathrm{D} \%$ & $\mathrm{~F} \%$ & $\mathrm{~N}$ & $\mathrm{D} \%$ & $\mathrm{~F} \%$ & $\mathrm{~N}$ & $\mathrm{D} \%$ & $\mathrm{~F} \%$ & $\mathrm{~N}$ & $\mathrm{D} \%$ & $\mathrm{~F} \%$ \\
\hline & Gastropoda & & & & & & & & & & & & \\
\hline MG & Alvania cancellata (da Costa, 1778) & - & - & - & - & - & - & 1 & 0.95 & 8.33 & - & - & - \\
\hline MG & Alvania cimex (Linnaeus, 1758) & - & - & - & - & - & - & 1 & 0.95 & 8.33 & - & - & - \\
\hline MG & Alvania discors (T. Allan, 1818) & - & - & - & 11 & 6.11 & 8.33 & 1 & 0.95 & 8.33 & 1 & 0.74 & 8.33 \\
\hline MG & Alvania geryonia (Nardo, 1847) & - & - & - & - & - & - & 2 & 1.90 & 16.67 & - & - & - \\
\hline MG & Alvania hirta (Monterosato, 1884) & - & - & - & - & - & - & 1 & 0.95 & 16.67 & - & - & - \\
\hline MG & Alvania hispidula (Monterosato, 1884) & - & - & - & - & - & - & 4 & 3.81 & 16.67 & - & - & - \\
\hline MG & Alvania lineata Risso, 1826 & - & - & - & 3 & 1.67 & 16.67 & 1 & 0.95 & 8.33 & 2 & 1.48 & 8.33 \\
\hline MG & Alvania mamillata Risso, 1826 & - & - & - & 1 & 0.56 & 8.33 & - & - & - & 1 & 0.74 & 8.33 \\
\hline MG & $\begin{array}{l}\text { Alvania pagodula (Bucquoy, Dautzenberg } \\
\text { \& Dollfus, 1884) }\end{array}$ & - & - & - & - & - & - & - & - & - & 5 & 3.70 & 16.67 \\
\hline MG & $\begin{array}{l}\text { Alvania subcrenulata (Bucquoy, } \\
\text { Dautzenberg \& Dollfus, 1884) }\end{array}$ & - & - & - & 19 & 10.56 & 25.00 & - & - & - & - & - & - \\
\hline MG & Ascobulla fragilis (Jeffreys, 1856) & - & - & - & - & - & - & - & - & - & 1 & 0.74 & 8.33 \\
\hline MG & Barleeia unifasciata (Montagu, 1803) & - & - & - & 5 & 2.78 & 25.00 & - & - & - & - & - & - \\
\hline MG & Bittium latreillii (Payraudeau, 1826) & - & - & - & 21 & 11.67 & 50.00 & 6 & 5.71 & 33.33 & 5 & 3.70 & 25.00 \\
\hline MG & Bittium lacteum (Philippi, 1836) & - & - & - & 1 & 0.56 & 8.33 & - & - & - & - & - & - \\
\hline MG & Bittium reticulatum (da Costa, 1778) & 7 & 1.97 & 16.67 & 6 & 3.33 & 33.33 & - & - & - & 1 & 0.74 & 8.33 \\
\hline $\mathrm{P}$ & Cerithiopsis sp. & - & - & - & 2 & 1.11 & 16.67 & - & - & - & - & - & - \\
\hline MG & Cerithium sp. & - & - & - & 1 & 0.56 & 8.33 & - & - & - & - & - & - \\
\hline$P$ & Chauvetia brunnea (Donovan, 1804) & - & - & - & - & - & - & - & - & - & 2 & 1.48 & 8.33 \\
\hline $\mathrm{P}$ & Chauvetia sp. & - & - & - & 1 & 0.56 & 8.33 & - & - & - & - & - & - \\
\hline MG & Columbella rustica (Linnaeus, 1758) & - & - & - & 13 & 7.22 & 50.00 & - & - & - & - & - & - \\
\hline$P$ & Coralliophila sp. & - & - & - & 2 & 1.11 & 16.67 & 1 & 0.95 & 8.33 & - & - & - \\
\hline $\mathrm{P}$ & Episcomitra cornicula (Linnaeus, 1758) & - & - & - & 4 & 2.22 & 16.67 & - & - & - & - & - & - \\
\hline $\mathrm{E}$ & Eulimella acicula (Philippi, 1836) & 1 & 0.28 & 8.33 & - & - & - & - & - & - & - & - & - \\
\hline$P$ & Fusinus pulchellus (Philippi, 1840) & - & - & - & - & - & - & 1 & 0.95 & 8.33 & - & - & - \\
\hline $\mathrm{P}$ & Fusinus sp. & - & - & - & - & - & - & - & - & - & 1 & 0.74 & 8.33 \\
\hline $\mathrm{P}$ & Gibberula philippii (Monterosato, 1878) & - & - & - & 1 & 0.56 & 8.33 & - & - & - & - & - & - \\
\hline$P$ & Gibberula recondita Monterosato, 1884 & - & - & - & 2 & 1.11 & 8.33 & - & - & - & - & - & - \\
\hline MG & Gibbula ardens (Salis Marschlins, 1793) & - & - & - & - & - & - & - & - & - & 2 & 1.48 & 16.67 \\
\hline MG & Haliotis sp. & - & - & - & - & - & - & - & - & - & 2 & 1.48 & 8.33 \\
\hline MG & Haminoea sp. & - & - & - & - & - & - & 2 & 1.90 & 8.33 & - & - & - \\
\hline
\end{tabular}




\begin{tabular}{|c|c|c|c|c|c|c|c|c|c|c|c|c|c|}
\hline \multirow[b]{2}{*}{ FG } & \multirow[b]{2}{*}{ species } & \multicolumn{3}{|c|}{ SB } & \multicolumn{3}{|c|}{ PHB } & \multicolumn{3}{|c|}{ SHB } & \multicolumn{3}{|c|}{ HP } \\
\hline & & $\mathrm{N}$ & $\mathrm{D} \%$ & $\mathrm{~F} \%$ & $\mathrm{~N}$ & $\mathrm{D} \%$ & $\mathrm{~F} \%$ & $\mathrm{~N}$ & $\mathrm{D} \%$ & $\mathrm{~F} \%$ & $\mathrm{~N}$ & $\mathrm{D} \%$ & $\mathrm{~F} \%$ \\
\hline $\mathrm{P}$ & Mangelia costulata Risso, 1826 & 1 & 0.28 & 8.33 & - & - & - & - & - & - & - & - & - \\
\hline $\mathrm{P}$ & Mangelia taeniata (Deshayes, 1835) & - & - & - & 1 & 0.56 & 8.33 & - & - & - & - & - & - \\
\hline MG & Manzonia crassa (Kanmacher, 1798) & - & - & - & 1 & 0.56 & 8.33 & - & - & - & - & - & - \\
\hline $\mathrm{E}$ & Melanella sp. & 1 & 0.28 & 8.33 & - & - & - & - & - & - & - & - & - \\
\hline $\mathrm{P}$ & Mitrella minor & - & - & - & 1 & 0.56 & 8.33 & - & - & - & - & - & - \\
\hline $\mathrm{P}$ & Muricopsis cristata (Brocchi, 1814) & - & - & - & - & - & - & 2 & 1.90 & 16.67 & - & - & - \\
\hline $\mathrm{P}$ & Neverita josephinia Risso, 1826 & 3 & 0.85 & 25.00 & - & - & - & - & - & - & - & - & - \\
\hline $\mathrm{P}$ & Ocenebra edwardsii (Payraudeau, 1826) & - & - & - & 1 & 0.56 & 8.33 & - & - & - & - & - & - \\
\hline $\mathrm{E}$ & Parthenina emaciata (Brusina, 1866) & - & - & - & 1 & 0.56 & 8.33 & - & - & - & - & - & - \\
\hline $\mathrm{P}$ & Pusia savignyi (Payraudeau, 1826) & - & - & - & 2 & 1.11 & 16.67 & 2 & 1.90 & 8.33 & - & - & - \\
\hline $\mathrm{P}$ & Pusia tricolor (Gmelin, 1791) & - & - & - & 5 & 2.78 & 33.33 & 2 & 1.90 & 16.67 & - & - & - \\
\hline MG & $\begin{array}{l}\text { Pusillina philippi (Aradas \& Maggiore, } \\
\text { 1844) }\end{array}$ & - & - & - & 3 & 1.67 & 25.00 & - & - & - & - & - & - \\
\hline MG & Pusillina sp. & - & - & - & 1 & 0.56 & 8.33 & 1 & 0.95 & 8.33 & - & - & - \\
\hline MG & Pusillina cf. radiata (Philippi, 1836) & - & - & - & - & - & - & - & - & - & 4 & 2.96 & 25 \\
\hline $\mathrm{E}$ & Pyrgiscus rufus (Philippi, 1836) & 3 & 0.85 & 8.33 & - & - & - & - & - & - & - & - & - \\
\hline $\mathrm{P}$ & Raphitoma bicolor (Risso, 1826) & - & - & - & - & - & - & - & - & - & 1 & 0.74 & 8.33 \\
\hline $\mathrm{P}$ & Raphitoma sp. & - & - & - & 1 & 0.56 & 8.33 & - & - & - & - & - & - \\
\hline MG & Rissoa auriscalpium (Linnaeus, 1758) & - & - & - & - & - & - & - & - & - & 6 & 4.44 & 25.00 \\
\hline MG & Rissoa rodhensis Verduin, 1985 & - & - & - & - & - & - & - & - & - & 1 & 0.74 & 8.33 \\
\hline MG & $\begin{array}{l}\text { Rissoa variabilis (Megerle von Mühlfeld, } \\
\text { 1824) }\end{array}$ & - & - & - & 1 & 0.56 & 8.33 & - & - & - & - & - & - \\
\hline MG & Rissoa violacea Desmarest, 1814 & - & - & - & - & - & - & - & - & - & 3 & 2.22 & 16.67 \\
\hline $\mathrm{P}$ & Roxania utriculus (Brocchi, 1814) & - & - & - & - & - & - & 1 & 0.95 & 8.33 & - & - & - \\
\hline MG & Smaragdia viridis (Linnaeus, 1758) & 1 & 0.28 & 8.33 & - & - & - & - & - & - & 15 & 11.11 & 50.00 \\
\hline MG & Tectura virginea (O. F. Müller, 1776) & - & - & - & 1 & 0.56 & 8.33 & - & - & - & - & - & - \\
\hline MG & Tricolia punctura Gofas, 1993 & - & - & - & - & - & - & - & - & - & 1 & 0.74 & 8.33 \\
\hline MG & Tricolia pullus (Linnaeus, 1758) & - & - & - & - & - & - & - & - & - & 1 & 0.74 & 8.33 \\
\hline MG & $\begin{array}{l}\text { Tricolia speciosa (Megerle von Mühlfeld, } \\
\text { 1824) }\end{array}$ & - & - & - & - & - & - & - & - & - & 8 & 5.93 & 33.33 \\
\hline $\mathrm{P}$ & Triphoridae & - & - & - & - & - & - & 4 & 3.81 & 25.00 & 2 & 1.48 & 16.67 \\
\hline $\mathrm{SC}$ & Tritia pellucida (Risso, 1826) & 8 & 2.25 & 33.33 & - & - & - & - & - & - & - & - & - \\
\hline $\mathrm{E}$ & Turbonilla acutissima Monterosato, 1884 & 3 & 0.85 & 16.7 & - & - & - & - & - & - & - & - & - \\
\hline $\mathrm{SF}$ & Turritella turbona Monterosato, 1877 & & & & - & - & - & 1 & 0.95 & 8.33 & - & - & - \\
\hline $\mathrm{P}$ & Volvulella acuminata (Bruguière, 1792) & 1 & 0.28 & 8.33 & - & - & - & - & - & - & - & - & - \\
\hline
\end{tabular}




\begin{tabular}{|c|c|c|c|c|c|c|c|c|c|c|c|c|c|}
\hline \multirow[b]{2}{*}{ FG } & \multirow[b]{2}{*}{ species } & \multicolumn{3}{|c|}{ SB } & \multicolumn{3}{|c|}{ PHB } & \multicolumn{3}{|c|}{ SHB } & \multicolumn{3}{|c|}{ HP } \\
\hline & & $\mathrm{N}$ & $\mathrm{D} \%$ & $\mathrm{~F} \%$ & $\mathrm{~N}$ & $\mathrm{D} \%$ & $\mathrm{~F} \%$ & $\mathrm{~N}$ & $\mathrm{D} \%$ & $\mathrm{~F} \%$ & $\mathrm{~N}$ & $\mathrm{D} \%$ & $\mathrm{~F} \%$ \\
\hline & Bivalvia & & & & & & & & & & & & \\
\hline SF & Acar clathrata (Defrance, 1816) & - & - & - & - & - & - & 2 & 1.90 & 16.67 & - & - & - \\
\hline SF & Arca noae Linnaeus, 1758 & - & - & - & - & - & - & 2 & 1.90 & 16.67 & - & - & - \\
\hline $\mathrm{DF}$ & Arcopella balaustina (Linnaeus, 1758) & - & - & - & - & - & - & 1 & 0.95 & 8.33 & - & - & - \\
\hline SF & Asperarca $\mathrm{sp}$. & - & - & - & - & - & - & 1 & 0.95 & 8.33 & - & - & - \\
\hline DF & Bosemprella incarnata (Linnaeus, 1758) & 1 & 0.28 & 8.33 & - & - & - & - & - & - & - & - & - \\
\hline SF & Cardita calyculata (Linnaeus, 1758) & - & - & - & 4 & 2.22 & 25.00 & 1 & 0.95 & 8.33 & - & - & - \\
\hline $\mathrm{SF}$ & Chama gryphoides Linnaeus, 1758 & - & - & - & - & - & - & - & - & - & 1 & 0.74 & 8.33 \\
\hline SF & Chamelea gallina (Linnaeus, 1758) & 4 & 1.13 & 33.33 & - & - & - & - & - & - & - & - & - \\
\hline SF & Chamidae & - & - & - & 1 & 0.56 & 8.33 & - & - & - & - & - & - \\
\hline DF & Ctena decussata (O. G. Costa, 1829) & - & - & - & - & - & - & - & - & - & 3 & 2.22 & 8.33 \\
\hline SF & Corbula gibba (Olivi, 1792) & 2 & 0.56 & 8.33 & - & - & - & - & - & - & - & - & - \\
\hline SF & Diplodonta trigona (Scacchi, 1835) & 7 & 1.97 & 25.00 & - & - & - & - & - & - & - & - & - \\
\hline SF & Donax venustus Poli, 1795 & 1 & 0.28 & 8.33 & - & - & - & - & - & - & - & - & - \\
\hline $\mathrm{SF}$ & Dosinia lupinus (Linnaeus, 1758) & 1 & 0.28 & 8.33 & - & - & - & - & - & - & - & - & - \\
\hline DF & Fabulina fabula (Gmelin, 1791) & 28 & 7.89 & 25.00 & - & - & - & - & - & - & - & - & - \\
\hline $\mathrm{SF}$ & Flexopecten hyalinus (Poli, 1795) & - & - & - & - & - & - & 1 & 0.95 & 8.33 & - & - & - \\
\hline SF & Galeomma turtoni W. Turton, 1825 & - & - & - & 1 & 0.56 & 8.33 & - & - & - & - & - & - \\
\hline $\mathrm{SF}$ & Glans trapezia (Linnaeus, 1767) & - & - & - & 1 & 0.56 & 8.33 & - & - & - & 28 & 20.74 & 66.67 \\
\hline $\mathrm{SF}$ & Glycymeris glycymeris (Linnaeus, 1758) & 1 & 0.28 & 8.33 & - & - & - & - & - & - & - & - & - \\
\hline SF & Gouldia minima (Montagu, 1803) & 1 & 0.28 & 8.33 & 1 & 0.56 & 8.33 & - & - & - & 6 & 4.44 & 25.00 \\
\hline $\mathrm{SF}$ & Gregariella semigranata (Reeve, 1858) & - & - & - & - & - & - & 4 & 3.81 & 33.33 & 1 & 0.74 & 8.33 \\
\hline SF & Hiatella arctica (Linnaeus, 1767) & - & - & - & - & - & - & 6 & 5.71 & 33.33 & 2 & 1.48 & 16.67 \\
\hline SF & Kurtiella bidentata (Montagu, 1803) & 2 & 0.56 & 8.33 & - & - & - & - & - & - & - & - & - \\
\hline $\mathrm{DF}$ & Lembulus pella (Linnaeus, 1758) & 4 & 1.13 & 25.00 & - & - & - & - & - & - & - & - & - \\
\hline $\mathrm{SF}$ & Limaria sp. & - & - & - & 5 & 2.78 & 16.67 & 2 & 1.90 & 16.67 & 2 & 1.48 & 8.33 \\
\hline SF & Lithophaga lithophaga (Linnaeus, 1758) & - & - & - & 3 & 1.67 & 16.67 & - & - & - & - & - & - \\
\hline SF & Loripinus fragilis (Philippi, 1836) & 11 & 3.10 & 25.00 & - & - & - & - & - & - & 1 & 0.74 & 8.33 \\
\hline SF & Lucinella divaricata (Linnaeus, 1758) & 156 & 43.94 & 83.33 & - & - & - & - & - & - & 1 & 0.74 & 8.33 \\
\hline SF & Mimachlamys varia (Linnaeus, 1758) & - & - & - & - & - & - & 1 & 0.95 & 8.33 & - & - & - \\
\hline SF & Modiolula phaseolina (Philippi, 1844) & - & - & - & - & - & - & 1 & 0.95 & 8.33 & - & - & - \\
\hline DF & Moerella donacina (Linnaeus, 1758) & 48 & 13.52 & 91.67 & - & - & - & - & - & - & - & - & - \\
\hline $\mathrm{DF}$ & Moerella pulchella (Lamarck, 1818) & 4 & 1.13 & 16.67 & - & - & - & - & - & - & - & - & - \\
\hline SF & Musculus costulatus (Risso, 1826) & - & - & - & 13 & 7.22 & 41.67 & 3 & 2.86 & 16.67 & 3 & 2.22 & 25.00 \\
\hline $\mathrm{SF}$ & Musculus subpictus (Cantraine, 1835) & - & - & - & 3 & 1.67 & 16.67 & 1 & 0.95 & 8.33 & 2 & 1.48 & 16.67 \\
\hline
\end{tabular}




\begin{tabular}{|c|c|c|c|c|c|c|c|c|c|c|c|c|c|}
\hline \multirow[b]{2}{*}{ FG } & \multirow[b]{2}{*}{ species } & \multicolumn{3}{|c|}{ SB } & \multicolumn{3}{|c|}{ PHB } & \multicolumn{3}{|c|}{ SHB } & \multicolumn{3}{|c|}{ HP } \\
\hline & & $\mathrm{N}$ & $\mathrm{D} \%$ & $\mathrm{~F} \%$ & $\mathrm{~N}$ & $\mathrm{D} \%$ & $\mathrm{~F} \%$ & $\mathrm{~N}$ & $\mathrm{D} \%$ & $\mathrm{~F} \%$ & $\mathrm{~N}$ & $\mathrm{D} \%$ & $\mathrm{~F} \%$ \\
\hline SF & Ostrea edulis Linnaeus, 1758 & - & - & - & - & - & - & 1 & 0.95 & 8.33 & - & - & - \\
\hline $\mathrm{SF}$ & Papillicardium papillosum (Poli, 1791) & - & - & - & - & - & - & 2 & 1.90 & 16.67 & 2 & 1.48 & 8.33 \\
\hline SF & $\begin{array}{l}\text { Parvicardium scriptum (Bucquoy, } \\
\text { Dautzenberg \& Dollfus, 1892) }\end{array}$ & - & - & - & 2 & 1.11 & 8.33 & - & - & - & - & - & - \\
\hline DF & Peronidia albicans (Gmelin, 1791) & 1 & 0.28 & 8.33 & - & - & - & - & - & - & - & - & - \\
\hline SF & Petricola lithophaga (Retzius, 1788) & - & - & - & - & - & - & - & - & - & 1 & 0.74 & 8.33 \\
\hline SF & Pitar rudis (Poli, 1795) & 2 & 0.56 & 8.33 & - & - & - & - & - & - & - & - & - \\
\hline SF & Polititapes aureus (Gmelin, 1791) & 3 & 0.85 & 25.00 & - & - & - & - & - & - & - & - & - \\
\hline $\mathrm{SF}$ & Pseudochama gryphina (Lamarck, 1819) & - & - & - & - & - & - & 1 & 0.95 & 8.33 & - & - & - \\
\hline $\mathrm{SF}$ & Rocellaria dubia (Pennant, 1777) & - & - & - & 1 & 0.56 & 8.33 & - & - & - & - & - & - \\
\hline SF & Solemya togata (Poli, 1791) & 1 & 0.28 & 8.33 & - & - & - & - & - & - & - & - & - \\
\hline SF & Spisula subtruncata (da Costa, 1778) & 4 & 1.13 & 16.67 & - & - & - & - & - & - & - & - & - \\
\hline $\mathrm{SF}$ & Spondylus gaederopus Linnaeus, 1758 & - & - & - & - & - & - & - & - & - & 1 & 0.74 & 8.33 \\
\hline SF & Striarca lactea (Linnaeus, 1758) & 1 & 0.28 & 8.33 & 18 & 10.00 & 58.33 & 30 & 28.57 & 58.33 & 15 & 11.11 & 50.00 \\
\hline SF & Talochlamys multistriata (Poli, 1795) & - & - & - & 1 & 0.56 & 8.33 & - & - & - & - & - & - \\
\hline SF & Tellimya ferruginosa (Montagu, 1808) & 1 & 0.28 & 8.33 & - & - & - & - & - & - & - & - & - \\
\hline SF & Thracia villosiuscula (MacGillivray, 1827) & 18 & 5.07 & 25.00 & - & - & - & 2 & 1.90 & 16.67 & - & - & - \\
\hline \multirow[t]{2}{*}{ SF } & Venus verrucosa Linnaeus, 1758 & - & - & - & - & - & - & - & - & - & 1 & 0.74 & 8.33 \\
\hline & Polyplacophora & & & & & & & & & & & & \\
\hline MG & Acanthochitona crinita (Pennant, 1777) & - & - & - & 4 & 2.22 & 25.00 & - & - & - & - & - & - \\
\hline MG & $\begin{array}{l}\text { Acanthochitona fascicularis (Linnaeus, } \\
\text { 1767) }\end{array}$ & - & - & - & 5 & 2.78 & 33.33 & - & - & - & 1 & 0.74 & 8.33 \\
\hline MG & Acanthochitona sp. & - & - & - & 1 & 0.56 & 8.33 & - & - & - & - & - & - \\
\hline MG & Callochiton septemvalvis (Montagu, 1803) & - & - & - & 4 & 2.22 & 25.00 & 7 & 6.67 & 25.00 & - & - & - \\
\hline \multirow[t]{2}{*}{ MG } & Chiton corallinus (Risso, 1826) & - & - & - & - & - & - & 2 & 1.90 & 8.33 & - & - & - \\
\hline & Scaphopoda & & & & & & & & & & & & \\
\hline DF & Fustiaria rubescens (Deshayes, 1825) & 20 & 5.63 & 25.00 & - & - & - & - & - & - & - & - & - \\
\hline DF & Dischides politus (S. Wood, 1842) & 3 & 0.85 & 8.33 & - & - & - & - & - & - & - & - & - \\
\hline $\mathrm{DF}$ & Antalis vulgaris (da Costa, 1778) & 1 & 0.28 & 8.33 & - & - & - & - & - & - & - & - & - \\
\hline
\end{tabular}

F 58.3\%), followed by the polyplacophoran Callochiton septemvalvis (DI 6.6\%; F 25\%) and gastropods belonging to Triphoridae family (DI $3.8 \%$; F $25 \%$ ).

In HP, gastropods were dominant in species richness (DQ $55.2 \%, 21 \mathrm{sp}$.), with an abundance of $47 \%$ (64 ind.), followed by bivalves (DQ 42.1\%, $16 \mathrm{sp}$.), with an abundance of $51.4 \%$ (70 ind.). The most abundant and frequent species were the gastropod Smaragdia viridis (DI 11.1\%; F 50\%) and the bivalve Glans trapezia (DI 20.7\%; F
66.6\%). The polyplacophoran $A$. fascicularis belongs to the third taxonomic group occurring in HP habitat, with a single individual (DQ 2.6\%, DI 0.3\%).

\subsection{Trophic diversity}

In the four MPAs six feeding guilds were identified (Fig. 3). Only three of them showed a high dominance: suspension feeders (SF) (DI 51.4\%; DQ 42.6\%), micro- and macro- 
grazers (MG) (DI 23.9\%; DQ 33.9\%) and deposit feeders (DF) (DI 14.6\%; DQ 6.4\%). The three remaining categories in total reached DI $9.9 \%$ and DQ $20.6 \%$.

The feeding guild SF was dominant in SHB habitat of

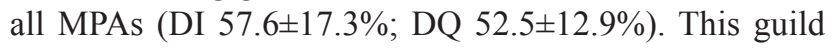
was mainly represented by the bivalve species $L$. divaricata $(157$ ind.) and $S$. lactea (64 ind.). The feeding guild MG was mostly dominant in both PHB (DI 51.9 $\pm 15.2 \%$; DQ

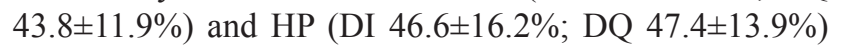
habitats. The main representative grazers were the gastropod species B. latreillii (32 ind.), A. subcrenulata (19 ind.) and $S$. viridis (16 ind.). The third dominant feeding guild, represented by DF, was dominant in SB habitat (DI $55.5 \pm 21.7 \%$; DQ $27.7 \pm 5.7 \%$ ) and was mainly repre- sented by the bivalve species $M$. donacina (total abundance of 48 ind.) and Fabulina fabula (28 ind.).

\subsection{Structural analysis}

Total number of individuals (N) per $\mathrm{m}^{-2}$, species richness (SR) and diversity indices (H', J) were reported in Table 2. The highest mean density among MPAs was observed in SB habitat, while the lowest occurred in SHB habitat. The highest and lowest mean species richness occurred in both hard bottoms, respectively in the photophilic (PHB) and sciaphilic (SHB) habitats. Both the diversity indices $\left(\mathrm{H}^{\prime}\right.$ and $\left.\mathrm{J}\right)$ resulted lower for SB than all the other habitats.
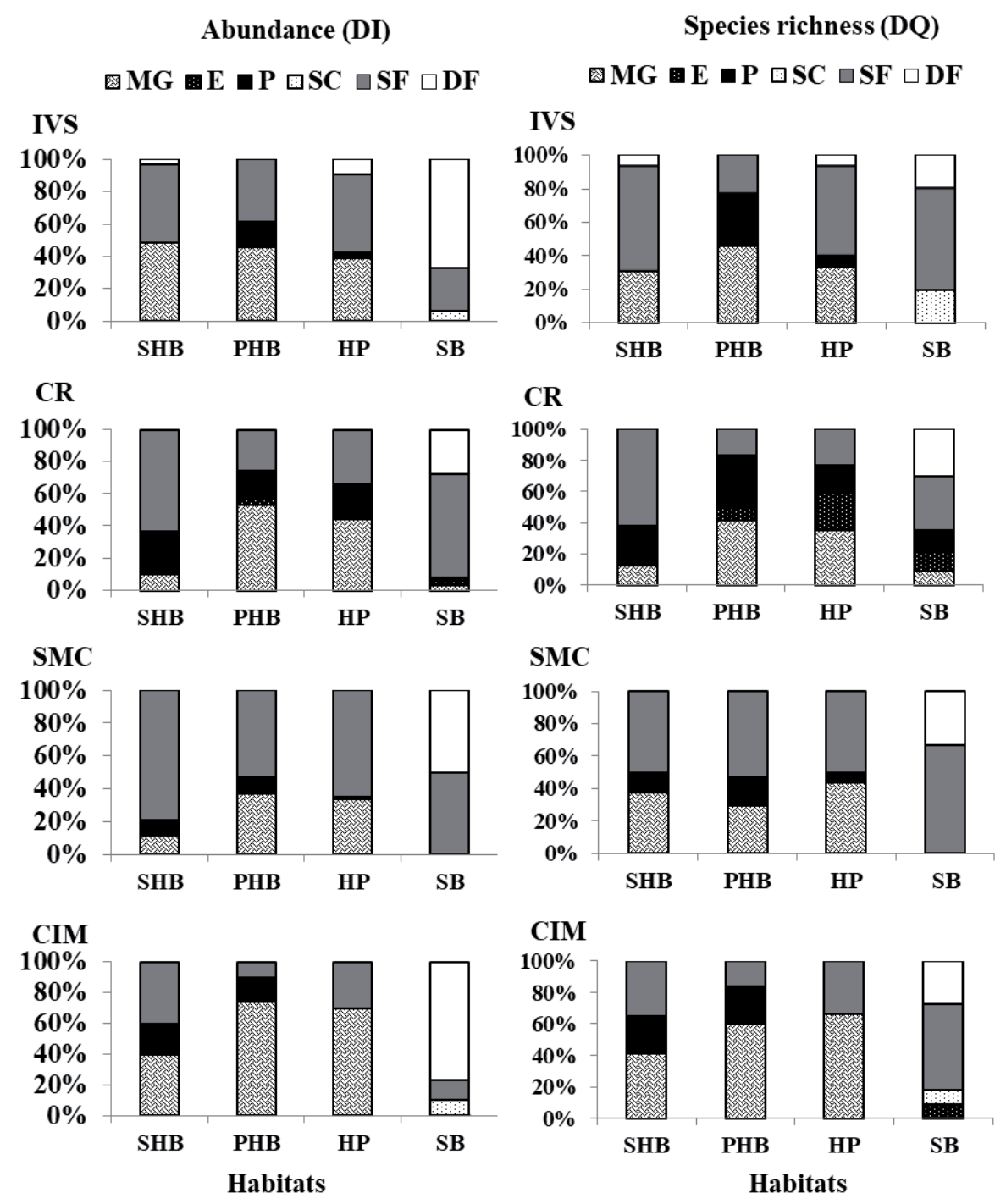

Fig. 3. Feeding-guilds (MG Micro- and Macro-grazers, E Ectoparasites, P Pre'dators, SC Scavengers, SF Suspension feeders, DF Detritus feeders) contribution to quantitative (DI) and qualitative (DQ) dominances for each habitat (SHB - sciaphilic hard bottom; PHB - photophilic hard bottom; HP - P. oceanica bed; SB - soft bottom;) and site (IVS - Isole di Ventotene and Santo Stefano; CR - Capo Rizzuto; SMC - Santa Maria di Castellabate; CIM - Costa degli Infreschi e della Masseta) 
Table 2. Mollusc assemblages: number of individuals per $\mathrm{m}^{-2}(\mathrm{~N})$, number of species (SR), and diversity indices (J, H') measured for each habitat (SB - soft bottom; PHB - photophilic hard bottom; SHB - sciaphilic hard bottom; HP - P. oceanica bed) among MPAs (mean \pm SD). Significance levels of PERMANOVA test: $*(\mathrm{p}<0.05), * *(\mathrm{p}<0.01) ; * * *(\mathrm{p}<0.001)$

\begin{tabular}{crccc}
\hline Habitats & N & SR & H' & J \\
\hline SB & $178.75 \pm 54.97^{* * *}$ & $6.75 \pm 1.35^{* *}$ & $1.81 \pm 0.33^{* *}$ & $0.73 \pm 0.05^{*}$ \\
PHB & $94.08 \pm 53.63^{*}$ & $7.83 \pm 3.15$ & $2.58 \pm 0.55$ & $0.93 \pm 0.03$ \\
SHB & $54.83 \pm 33.44$ & $5.58 \pm 2.24$ & $2.13 \pm 0.49$ & $0.90 \pm 0.06$ \\
HP & $71.08 \pm 26.91$ & $6.16 \pm 1.82$ & $2.38 \pm 0.41$ & $0.94 \pm 0.02$ \\
\hline
\end{tabular}

PERMANOVA test (Table 2) highlighted significant differences of mollusc density, species richness and diversity indices among the SB of the four MPAs. A significant difference of mollusc density was also observed among the PHB sampled in the four MPAs.

In all MPAs, the composition and structure of mollusc assemblages among the four habitats was significantly different $(\mathrm{F}=8.078, \mathrm{p}=0.0002, \mathrm{df}=3)$. Therefore, significant differences were also detected by comparing the four MPAs $(\mathrm{F}=3.317, \mathrm{p}=0.0002, \mathrm{df}=3)$, so as interactions between habitats and sites (MPAs) $(\mathrm{F}=2.294, \mathrm{p}=0.0002$, $\mathrm{df}=9$ ). Pairwise comparisons of habitats (Table 3 ) showed that, in all sites, SB was significantly different from the other three habitats (PHB, SHB, and HP). Significant dif-

Table 3. Results of PERMANOVA pairwise comparisons among the habitats (SB - soft bottom; PHB - photophilic hard bottom; SHB - sciaphilic hard bottom; HP - P. oceanica bed) for each site (IVS - Isole di Ventotene and Santo Stefano; SMC - Santa Maria di Castellabate; CIM Costa degli Infreschi e della Masseta; CR - Capo Rizzuto), using 4999 permutations

\begin{tabular}{ccccc} 
& \multicolumn{4}{c}{ Sites } \\
\cline { 2 - 5 } Habitats & ISV & CR & SMC & CIM \\
\hline & $\mathrm{t}-p$ & $\mathrm{t}-p$ & $\mathrm{t}-p$ & $\mathrm{t}-p$ \\
$\mathrm{SB}-\mathrm{PHB}$ & $3.53^{* *}$ & $2.89 * *$ & $2.64^{*}$ & $2.05^{*}$ \\
$\mathrm{SB}-\mathrm{SHB}$ & $1.98^{*}$ & $2.09^{*}$ & $3.37^{* *}$ & $1.83^{*}$ \\
$\mathrm{SB}-\mathrm{HP}$ & $2.11^{*}$ & $2.04^{*}$ & $3.30^{* *}$ & $3.76^{* *}$ \\
$\mathrm{PHB}-\mathrm{SHB}$ & n.s. & n.s. & $1.87^{*}$ & n.s. \\
$\mathrm{PHB}-\mathrm{HP}$ & n.s. & n.s. & n.s. & $2.01 *$ \\
$\mathrm{SHB}-\mathrm{HP}$ & n.s. & n.s. & $2.14^{*}$ & n.s. \\
\hline
\end{tabular}

Significance levels: n.s. $(p>0.05), *(p<0.05)$, ** $(p<0.01) ; * * *(p<0.001)$. ferences were also detected at SMC site, between SHB on one side and PHB and HP on the other side $(p<0.05)$. Further significant differences were also found at CIM site, between PHB and HP $(p<0.05)$.

Pairwise comparisons of MPAs (Table 4) showed significant differences between $\mathrm{CR}$ and all the other three sites, as for the SB habitat $(p<0.01)$. Significant differences were also detected between CR on one side and IVS and SMC on the other side, for the PHB habitat $(p<0.05)$, and between CIM on one side and IVS and SMC on the other, for the HP habitat $(p<0.05)$. Significant differences among the four sites for the SHB habitat were not detected.

The differences in structure of mollusc assemblages were evident in the CAP plot (Fig. 4).

Table 4. Results of PERMANOVA pairwise comparisons among the sites (IVS - Isole di Ventotene and Santo Stefano; SMC - Santa Maria di Castellabate; CIM - Costa degli Infreschi e della Masseta; CR - Capo Rizzuto) for each habitat (SB - soft bottom; PHB - photophilic hard bottom; SHB - sciaphilic hard bottom; HP - P. oceanica bed), using 4999 permutations

\begin{tabular}{ccccc} 
& \multicolumn{4}{c}{ Habitats } \\
\cline { 2 - 5 } Sites & $\mathrm{SB}$ & $\mathrm{PHB}$ & $\mathrm{SHB}$ & $\mathrm{HP}$ \\
\hline t- $p$ & $\mathrm{t}-p$ & $\mathrm{t}-p$ & $\mathrm{t}-p$ \\
IVS - CR & $3.66^{* *}$ & $2.19^{*}$ & n.s. & n.s. \\
IVS - SMC & n.s. & n.s. & n.s. & n.s. \\
IVS - CIM & n.s. & n.s. & n.s. & $2.07^{*}$ \\
CR - SMC & $3.87^{* *}$ & $2.06^{*}$ & n.s. & n.s. \\
CR - CIM & $3.01^{* *}$ & n.s. & n.s. & n.s. \\
SMC - CIM & $2.02^{*}$ & n.s. & n.s. & $1.99 *$ \\
\hline
\end{tabular}

Significant levels: n.s. $(p>0.05), *(p<0.05)$, $* *(p<0.01) ; * * *(p<0.001)$. 
a

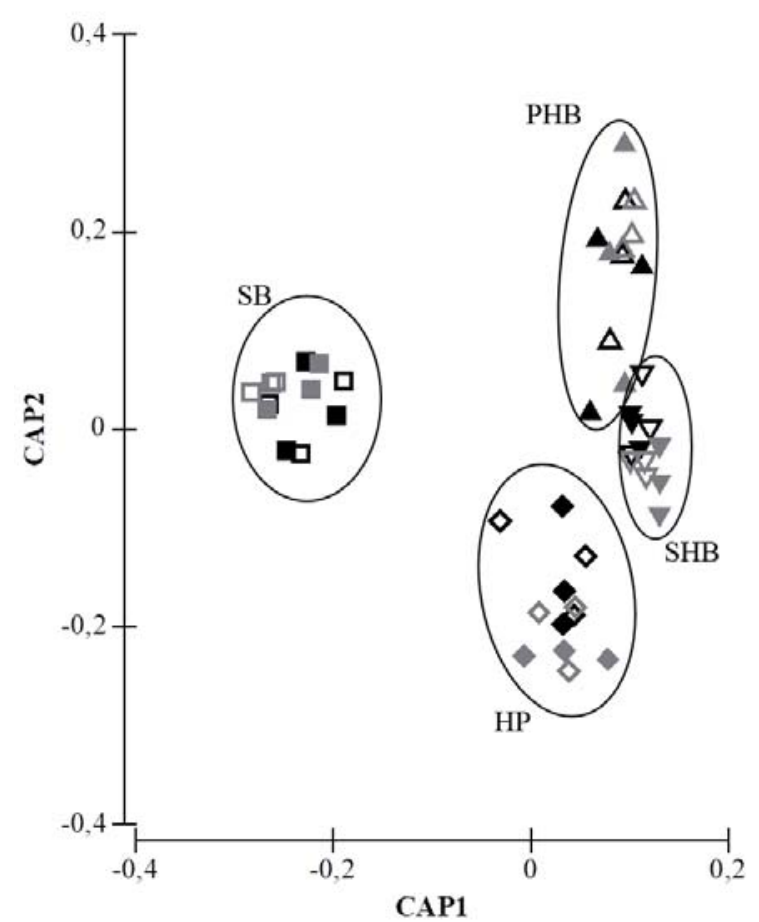

b

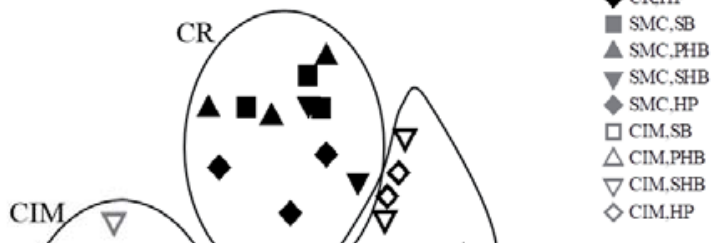

Fig. 4. CAP plot showing spatial distribution of mollusc assemblages for the habitat factor (a) (SB - soft bottom; PHB - photophilic hard bottom; SHB - sciaphilic hard bottom; HP - P. oceanica bed) and the site factor (b) (CIM - Costa degli Infreschi e della Masseta; CR - Capo Rizzuto; IVS - Isole di Ventotene and Santo Stefano; SMC - Santa Maria di Castellabate)

As for the habitat factor (Fig. 4a), the total sample points of SB form a cluster apart from all other habitats, clearly separated along CAP1. The other sample points were displaced along CAP2, forming a gradient from photophilic bottoms, with positive saturations, sciaphilic bottoms, with intermediate saturations, to seagrass beds, with negative saturations.

Instead, as for the MPA factor (Fig 4b), the sample points of CIM form a cluster apart from the all other MPAs, clearly separated along CAP1. The other sample points were displaced along CAP2, forming a gradient from CR, with positive saturations, IVS with intermediate saturations, to SMC with negative saturations. In all MPAs the most widespread replicate points were those of PHB, according to a higher variability in the mollusc taxocoene.

The SIMPER analysis (Table 5) showed that the highest similarity of dominant species among the samples taken in the four MPAs occurred for the SB habitat (average value of $36.51 \%$ ), where the species that mostly contributed to this similarity were at all sites the deposit feeder $M$. donacina and the suspension feeder L. divaricata. In HP (average value of 18.55\%) and PHB (average value of
$18.04 \%$ ) habitats, the species that are mostly responsible for the similarity at the assemblages found in MPAs were respectively the suspension feeders S. lactea and Glans trapezia, and the micrograzer $B$. latreillii. The lowest similarity was observed in the SHB (average value of $13.86 \%$ ), where $S$. lactea and B. latreillii were the most abundant species.

As for the trophic structure, the PERMANOVA analysis showed that the assemblages significantly differed among habitats $(\mathrm{F}=19.95, p<0.001)$. Significant differences were also present by comparing the trophic structure among the four MPAs $(\mathrm{F}=2.98, p=0.002)$, while the interaction between habitats and sites was not significant $(\mathrm{F}=1.61, p=0.059)$.

The ITD analysis showed that the habitats with the highest trophic diversity were PHB and HP, with a mean value respectively of $0.604 \pm 0.06$ and $0.549 \pm 0.12$, followed by SHB and SB, with a mean value respectively of $0.516 \pm 0.12$ and $0.365 \pm 0.08$. 
Table 5. Results of similarity of percentages test, showing species that mostly contributed to similarity of the samples for each habitat (SB - soft bottom; PHB - photophilic hard bottom; HP - P. oceanica bed; SHB - sciaphilic hard bottom)

\begin{tabular}{|c|c|c|c|c|c|c|}
\hline & & & & & & \\
\hline & Species & Av.Abund & Av.Sim & $\mathrm{Sim} / \mathrm{SD}$ & Contrib\% & Cum.\% \\
\hline \multirow{5}{*}{$\begin{array}{l}\text { SB - average similarity: } \\
36.51 \%\end{array}$} & Moerella donacina & 2.81 & 18.59 & 1.34 & 50.92 & 50.92 \\
\hline & Lucinella divaricata & 3.28 & 11.39 & 1.24 & 31.18 & 82.1 \\
\hline & Chamelea gallina & 0.66 & 1.47 & 0.31 & 4.04 & 86.14 \\
\hline & Tritia pellucida & 0.76 & 1.37 & 0.31 & 3.76 & 89.9 \\
\hline & Diplodonta trigona & 0.62 & 0.9 & 0.22 & 2.46 & 92.36 \\
\hline \multirow{8}{*}{$\begin{array}{l}\text { HP - average similarity: } \\
18.55 \%\end{array}$} & Glans trapezia & 1.94 & 6.93 & 0.83 & 37.35 & 37.35 \\
\hline & Smaragdia viridis & 1.34 & 3.14 & 0.53 & 16.95 & 54.3 \\
\hline & Striarca lactea & 1.35 & 2.81 & 0.53 & 15.15 & 69.45 \\
\hline & Tricolia speciosa & 0.85 & 1.44 & 0.31 & 7.75 & 77.2 \\
\hline & Bittium latreillii & 0.58 & 0.68 & 0.21 & 3.69 & 80.89 \\
\hline & Pusillina cf. radiata & 0.55 & 0.68 & 0.21 & 3.66 & 84.54 \\
\hline & Gouldia minima & 0.63 & 0.65 & 0.22 & 3.49 & 88.04 \\
\hline & Musculus costulatus & 0.5 & 0.55 & 0.21 & 2.98 & 91.02 \\
\hline \multirow{11}{*}{$\begin{array}{l}\text { PHB - average } \\
\text { similarity: } 18.04 \%\end{array}$} & Striarca lactea & 1.57 & 3.63 & 0.65 & 20.11 & 20.11 \\
\hline & Bittium latreillii & 1.43 & 2.91 & 0.53 & 16.12 & 36.23 \\
\hline & Columbella rustica & 1.31 & 2.44 & 0.52 & 13.55 & 49.78 \\
\hline & Musculus costulatus & 1.14 & 1.87 & 0.41 & 10.36 & 60.15 \\
\hline & Bittium reticulatum & 0.76 & 1.49 & 0.31 & 8.26 & 68.4 \\
\hline & Acanthochitona fascicularis & 0.71 & 0.87 & 0.3 & 4.81 & 73.21 \\
\hline & Pusia tricolor & 0.71 & 0.81 & 0.3 & 4.48 & 77.7 \\
\hline & Acanthochitona crinita & 0.55 & 0.68 & 0.21 & 3.78 & 81.48 \\
\hline & Alvania subcrenulata & 0.93 & 0.58 & 0.21 & 3.21 & 84.69 \\
\hline & Callochiton septemvalvis & 0.55 & 0.56 & 0.19 & 3.11 & 87.8 \\
\hline & Cardita calyculata & 0.55 & 0.45 & 0.22 & 2.52 & 90.32 \\
\hline \multirow{9}{*}{$\begin{array}{l}\text { SHB - average } \\
\text { similarity: } 13.86 \%\end{array}$} & Striarca lactea & 1.74 & 5.73 & 0.61 & 41.34 & 41.34 \\
\hline & Bittium latreillii & 0.74 & 2.15 & 0.31 & 15.47 & 56.81 \\
\hline & Gregariella semigranata & 0.66 & 1.47 & 0.29 & 10.62 & 67.44 \\
\hline & Hiatella arctica & 0.74 & 1.03 & 0.31 & 7.4 & 74.83 \\
\hline & Triphoridae & 0.55 & 0.74 & 0.22 & 5.35 & 80.19 \\
\hline & Callochiton septemvalvis & 0.62 & 0.62 & 0.21 & 4.45 & 84.63 \\
\hline & Arca noae & 0.33 & 0.32 & 0.12 & 2.3 & 86.93 \\
\hline & Pusia tricolor & 0.33 & 0.3 & 0.12 & 2.19 & 89.12 \\
\hline & Muricopsis cristata & 0.33 & 0.25 & 0.12 & 1.82 & 90.94 \\
\hline
\end{tabular}




\section{Discussion}

Four habitats have been taken into account: $P$. oceanica seagrass beds, hard bottoms with photophilic or sciaphilic coverage, and soft bottoms, mainly represented by sandy substrate. Results revealed marked differences in mollusc assemblages among the investigated habitats in four MPAs located along a latitudinal gradient of the Italian shores. Even though the MPAs are at a considerable distance from each other, from the Tyrrhenian to the Ionian basin, a biogeographical variability on the mollusc assemblages was not evident.

The highest species richness and trophic diversity were detected in photophilic hard bottom, which in turn showed a lowest number of individuals compared to the other habitats. In contrast, the soft bottom, even though characterized by the lowest species richness and trophic diversity, showed the highest number of individuals. This inverse relationship between species richness and abundance is rather frequent in ecological systems. In fact, the most accepted hypothesis is that higher is the number of species, smaller is the dimension of their populations due to the resource partitioning (Schoener, 1974; Brown, 1984).

The most abundant species found in the photophilic habitat belongs to different feeding guilds, and were mainly represented by grazers, suspension feeders and carnivores; this explains the highest value of trophic diversity index $(0.604 \pm 0.06)$, and it is due to the habitat heterogeneity effect (Tews et al., 2004). Macroalgae are the main component of the cover in photophilic hard bottom and provide an excellent substratum for microalgae (e.g. diatoms). In this habitat, grazers were mainly represented by the gastropods B. latreillii, Bittium reticulatum and Columbella rustica; other herbivore molluscs were the polyplacophorans A. fascicularis, Acanthochitona crinita and C. septemvalvis. This diversity in herbivore grazer species could be explained by the number of the different available food sources. Indeed, Chemello and Russo (1997) and Chemello and Milazzo (2002), pointed out that different types of macroalgae with their complex and variate architecture promote both abundance and diversity in the molluscan assemblages. The hard substrate would also enhance the presence of suspension feeders, as M. costulatus and S. lactea, which anchor themselves to the bottom through byssus threads (Hrs-Brenko and Legac, 2006).

A rather high trophic diversity index $(0.549 \pm 0.12)$ was also detected in P. oceanica seagrass beds. Mazzella et al. (1992) reported that in the photophilic layer, formed by the plant's leaves and their epiphytes, the high vegetable availability explains the high abundance of herbivores. Coherently, in this study micrograzers as B. latreillii, S. viridis, and $T$. speciosa strongly characterized seagrass beds in all the four MPAs. Beyond the herbivore gastropods of leaf stratums, SIMPER analysis also selected some bivalve species (G. trapezia, S. lactea, M. costulatus and G. minima) as important in structuring mollusc assemblages of the $P$. oceanica beds in the four MPAs. This is due to the contribution to the assemblages of species living in the rhizome's stratum, at the base of the plant, colonized by sciaphilic coralline algae and covered by sandy-muddy sediments (Orth et al., 1984; Mazzella et al., 1995).

Boudouresque et al. (1981) pointed out that even if a high number of sciaphilic algae species could be detected on Posidonia rhizomes, these are not characteristic of such habitat but their presence is simply due to the low light condition. Therefore, such algal assemblages can also be found in other sciaphilic habitats as those of hard bottoms. In fact, some mollusc species found both in photophilic hard bottom and seagrass bed habitats (e.g. B. latreillii, S. lactea and M. costulatus, together with Hiatella arctica and Gregariella semigranata) were also found in the sciaphilic hard bottom. This latter habitat showed a lower trophic diversity index $(0.516 \pm 0.12)$ due to the dominance in the mollusc assemblage of suspension feeders (about $58 \%$ ). This result, found for all the four investigated MPAs, strongly agrees with Gili and Coma (1998) who stressed that the species belonging to this trophic group are the dominant components of the coralligenous community of the Mediterranean Sea. According to the present study, this is true not only for sessile animal species but also for sedentary and vagile molluscs.

The PERMANOVA analysis pointed out significant differences in mollusc species composition among the photophilic, seagrass and sciaphilic habitats. Yet, the SIMPER test also underlined common elements among these three habitats due to the co-presence of few very abundant species.

The mollusc assemblage of sandy bottom was totally different from those living in the three previous habitats and this is particularly evident in the CAP plot. The lowest trophic diversity index of $0.365 \pm 0.08$ was detected for this habitat. This is due to the very high dominance of two feeding guilds: deposit feeders (about 56\%) and suspension feeders (about 38\%). The most abundant species found in the soft bottoms of the four MPAs were the burrowing deposit feeder $M$. donacina, which sucks organic matter on the sediment by its long extensive siphon, and the suspension feeder $L$. divaricata, which obtains approximately $40 \%$ of its metabolic requirements from gill endosymbionts (Le Pennec et al., 1995; Dey, 2006). These bivalves are among benthic species defined by Simboura and Zenetos (2002) as "sensitive indicators", well adapted to fine sands, the type of sediment more widespread in the four MPAs. 


\section{Conclusions}

At present, some peculiar habitats, such as $P$. oceanica beds and coralligenous formations, are considered of priority interest for conservation and protected by European Habitat Directive (92/43/EEC) as they are key habitats for a huge variety of associated species. The present results highlighted an important mollusc diversity not only in the well-known priority key habitats but also in those less investigated and considered in relation to nature conservation and more exposed to human pressure, such as photophilic hard bottom. Their status may be a good indicator of effective management and preservation of life in MPAs.

Several authors recognized molluscs among the major components in the biodiversity of different marine habitats, through which the environmental state could be evaluated (Sánchez-Moyano, 2000; Salánki et al., 2003; Arribas et al., 2014; Dimitriadis et al., 2014). Therefore, the assessment of mollusc assemblages can represent a useful tool for the management of coastal environments. In fact, changes in structures of mollusc assemblages can indicate fast response to changes in environmental conditions.

Finally, we conclude that habitats characterized by a well-structured molluscan assemblage can indicate a good quality status of MPAs.

\section{References}

Anderson M. J., 2001a, A new method for non-parametric multivariate analysis of variance, Austral Ecology, 26: 32-46.

Anderson M. J., 2001b, Permutation tests for univariate or multivariate analysis of variance and regression, Canadian Journal of Fisheries and Aquatic Sciences, 58(Supplement 3): 626-639.

Anderson M. J., Gorley R. N., Clarke K. R., 2008, PERMANOVA+ for PRIMER: Guide to Software and Statistical Methods. Plymouth.

Anderson M. J., Willis T. J., 2003, Canonical analysis of principal coordinates: A useful method of constrained ordination for ecology, Ecology, 84(Supplement 2): 511-525.

Antit M., Daoulatli A., Urra .J, Rueda J. L., Gofas S., Salas C., 2016, Seasonality and trophic diversity in molluscan assemblages from the Bay of Tunis (southern Mediterranean Sea), Mediterranean Marine Science, 17(Supplement 3): 692-707.

Antoniadou C., Chintiroglou C., 2005, Biodiversity of zoobenthic hard-substrate sublittoral communities in the Eastern Mediterranean (North Aegean Sea), Estuarine, Coastal and Shelf Science, 62(Supplement 4): 637-653.
Appolloni L., Bevilacqua S., Sbrescia L., Sandulli R., Terlizzi A., Russo G. F., 2017, Does full protection count for the maintenance of $\beta$-diversity patterns in marine communities? Evidence from Mediterranean fish assemblages, Aquatic Conservation: Marine and Freshwater Ecosystems, 27(Supplement 4): 828-838.

Appolloni L., Sandulli R., Vetrano G., Russo G. F., 2018, Assessing the effects of habitat patches ensuring propagule supply and different costs inclusion in marine spatial planning through multivariate analyses, Journal of Environmental Management, 214: 45-55.

Arribas L. P., Donnarumma L., Palomo M. G., Scrosati R. A., 2014, Intertidal mussels as ecosystem engineers: Their associated invertebrate biodiversity under contrasting wave exposures, Marine Biodiversity, 44(Supplement 2): 203-211.

Arruda E. P., Domaneschi O., Amaral A. C. Z., 2003, Mollusc feeding guilds on sandy beaches in São Paulo State, Brazil, Marine Biology, 143(Supplement 4): 691-701.

Ballesteros E. 2006, Mediterranean coralligenous assemblages: a synthesis of present knowledge, Oceanography and Marine Biology: an annual review, 44: 123195.

Bianchi C. N., Pronzato R., Cattaneo-Vietti R., BenedettiCecchi L., Morri C., Pansini M., Chemello R., Milazzo M., Fraschetti S., Terlizzi A., Peirano A., Salvati E., Benzoni F., Calcinai B., Cerrano C., Bavestrello G., 2004, Hard bottoms. Biol Mar Mediterr, 11(Supplement 1): 185-215.

Bouchet P., 2006, The magnitude of marine biodiversity, [in:] The Exploration of Marine Biodiversity: Scientific and Technological Challenges, C. M. Durante (ed), Fundación BBVA: 31-62.

Boudouresque C. F., Cinelli F., Fresi E., Mazzella L., Richard M., 1981, Algal undergrowth of Posidonia oceanica beds in the Gulf of Naples: Floristic study. Rapp. Comm. Int. Mer. Médit. 27: 195-196.

Browman H. I., Cury P. M., Hilborn R., Jennings S., Lotze H. K., Mace P. M., Murawski S., Pauly D., Sissenwine M., Stergiou K. I., Zeller D., 2004, Perspectives on ecosystem-based approaches to the management of marine resources, Marine Ecology Progress Series, 274: 269303.

Brown J. H., 1984, On the relationship between abundance and distribution of species, The American Naturalist, 124(Supplement 2): 255-279.

Casellato S., Stefanon A., 2008, Coralligenous habitat in the northern Adriatic Sea: An overview, Marine Ecology, 29(Supplement 3): 321-341.

Cattaneo-Vietti R., Russo G. F., 1987, Mollusc from submarine caves of the Sorrentine Peninsula (Southern Tyrrhenian Sea), Stygologia, 3(Supplement 2): 138148. 
Chemello R., Ciuna I., Pandolfo A., Riggio S., 1997, Molluscan assemblage associated with intertidal vermetid formations: a morpho-functional approach, Bollettino Malacologico, 33: 105-114.

Chemello R., Milazzo M., 2002, Effect of algal architecture on associated fauna: Some evidence from phytal molluscs, Marine Biology, 140(Supplement 5): 981-990.

Chemello R., Russo G. F. 1997, The molluscan taxocoene of photophilic algae from the Island of Lampedusa (Strait of Sicily, southern Mediterranean), Bollettino Malacologico, 33: 95-104.

Cicin-Sain B., Belfiore S., 2005, Linking marine protected areas to integrated coastal and ocean management: a review of theory and practice, Ocean \& Coastal Management, 48(Supplement 11-12): 847-868.

Clarke K. R., Gorley R. N., 2006, PRIMER v.6: User Manual/Tutorial. Plymouth.

Clarke K. R., Warwick R. M., 2001, Change in marine communities: an approach to statistical analysis and interpretation. Prim. Plymouth.

Coll M., Piroddi C., Steenbeek J., Kaschner K., Ben F., Lasram R., Ballesteros E., Bianchi C. N., Corbera J., Dailianis T., Kesner-reyes K., Kitsos M., Rius-barile J., Martin D., Mouillot D., Oro D., Villanueva R., Voultsiadou E., 2010, The Biodiversity of the Mediterranean Sea: Estimates, Patterns, and Threats, PLoS One, 5: e11842.

Dey A., 2006, Contribution to the knowledge of Indian Marine Molluscs (Part-IV). Family Tellinidae, Rec. Zool. Surv. India, 249: 1-124.

Díaz S., Cabido M., 2001, Vive la différence: plant functional diversity matters to ecosystem processes, Trends in Ecology \& Evolution, 16(Supplement 11): 646-655.

Dimitriadis C., Koutsoubas D., Garyfalou Z., Tselepides A., 2014, Benthic molluscan macrofauna structure in heavily trawled sediments (Thermaikos Gulf, North Aegean Sea): Spatiotemporal patterns, Journal of Biological Researc-Thessaloniki, 21(Supplement 1): 10.

Donnarumma L., Bruno R., Terlizzi A., Russo G. F., 2016, Population ecology of Gibbula umbilicaris and Gibbula ardens (Gastropoda: Trochidae) in a Posidonia oceanica seagrass bed, Italian Journal of Zoology, 83: 103-112.

Donnarumma L., Bruno R., Terlizzi A., Russo G. F., 2018, Population ecology of Jujubinus striatus and Jujubinus exasperatus (Gastropoda: Trochidae) in a Posidonia oceanica seagrass bed, The European Zoological Journal, 85: 17-25.

Donnarumma L., Sandulli R., Appolloni L., Di Stefano F., Russo G. F., 2018b, Morpho-structural and ecological features of a shallow vermetid bioconstruction in the Tyrrhenian Sea (Mediterranean Sea, Italy), Journal of Sea Research, 131: 61-68.
Fenberg P. B., Caselle J. E., Claudet J., Clemence M., Gaines S. D., García-Charton A. J., Gonçalves E. J., Grorud-Colvert K., Guidetti P., Jenkins S. R., Jones P. J. S., Lester S. E., McAllen R., Moland E., Planes S., Sørensen T. K., 2012, The science of European marine reserves: Status, efficacy, and future needs, Marine Policy, 36(Supplement 5): 1012-1021.

Ferrigno F., Appolloni L., Russo G. F., Sandulli R., 2018, Impact of fishing activities on different coralligenous assemblages of Gulf of Naples (Italy), Journal of the Marine Biological Association of the United Kingdom, 98: 41-50.

Ferrigno F., Russo G. F., Sandulli R., 2017, Coralligenous Bioconstructions Quality Index (CBQI): a synthetic indicator to assess the status of different types of coralligenous habitats, Ecological Indicators, 82: 271-279.

Franzese P. P., Buonocore E., Donnarumma L., Russo G. F., 2017, Natural capital accounting in marine protected areas: The case of the Islands of Ventotene and S. Stefano (Central Italy), Ecological Modelling, 360: 290-299.

Franzese P. P., Buonocore E., Paoli C., Massa F., Donati S., Fanciulli G., Miccio A., Mollica E., Navone A., Russo G. F., Povero P., Vassallo P., 2015, Environmental Accounting in Marine Protected Areas: the EAMPA Project, Journal of Environmental Accounting and Management 3(Supplement 4): 324-332.

Franzese P. P., Russo G. F., Ulgiati S., 2008, Modelling the interplay of environment, economy and resources in Marine Protected Areas. A case study in Southern Italy, Ecological Questions 1: 91-97.

Fretter V., Graham A., 1994, British prosobranch molluscs. Their functional anatomy and ecology, The Ray Society: London.

Gacia E., Costalago D., Prado P., Piorno D., Tomas F., 2009, Mesograzers in Posidonia oceanica meadows: An update of data on gastropod-epiphyte-seagrass interactions, Botanica Marina, 52(Supplement 5): 439447.

Gili J. M., Coma R., 1998, Benthic suspension feeders: their paramount role in littoral marine food webs, Trends in ecology \& evolution, 13(Supplement 8): 316-321.

Gladstone W., 2002, The potential value of indicator groups in the selection of marine reserves, Biological Conservation, 104(2): 211-220.

Guidetti P., Milazzo M., Bussotti S., Molinari A., Murenu M., Pais A., Spanò N., Balzano R., Agardy T., Boero F., Carrada G., Cattaneo-Vietti R., Cau A., Chemello R., Greco S., Manganaro A., Notarbartolo di Sciara G., Russo G. F., Tunesi L., 2008, Italian marine reserve effectiveness: Does enforcement matter? Biological Conservation, 141(Supplement 3): 699-709.

Halpern B. S., Lester S. E., McLeod K. L., 2010, Placing marine protected areas onto the ecosystem-based man- 
agement seascape, Proceedings of the National Academy of Sciences, 107(Supplement 43): 18312-18317.

Heip C. H. R., Goosen N. K., Herman P. M. J., Kromkamp J., Middelburg J. J., Soetaert K., 1995, Production and consumption of biological particles in temperate tidal estuaries, Oceanography and Marine Biology: an annual review, 33: 1-149.

Help C. H. R., Herman P. M. J., Soetaert K. 1998, Indices of diversity and evenness, Oceanis, 24(Supplement 6): 61-88.

Hemminga M. A., Duarte C. M., 2000, Seagrass ecology, Cambridge University Press.

Hrs-Brenko M, Legac M., 2006, Inter-and intra-species relationships of sessile bivalves on the eastern coast of the Adriatic Sea, Natura Croatica, 15(Supplement 4): 203-230.

Katsanevakis S., Stelzenmüller V., South A., Sørensen T. K., Jones P. J., Kerr S., Badalamenti F., Anagnostou C., Breen p., Chusth G., D’Anna G., Duijn M., Filatova T., Fiorentino F., Hulsman H., Johnson K., Karageorgis A. P., Kröncke I., Mirto S., Pipitone C., Portelli S., Qiu W., Reiss H., Sakellariou D., Salomidi M., Hoof L., Vassilopoulou V., Fernández T. V., Vöge S., Weber A., Zenetos A., ter Hofstede R., 2011, Ecosystem-based marine spatial management: review of concepts, policies, tools, and critical issues, Ocean \& Coastal Management, 54(Supplement 11): 807-820.

Lastra M., Palacio J., Sanchez A., Mora J., 1991, Estructura trófica infralitoral de la bahía de Santander, Cahiers de Biologie Marine, 32(Supplement 3): 333-351.

Le Pennec M., Beninger P. G., Herry A., 1995, Feeding and digestive adaptations of bivalve molluscs to sulphiderich habitats, Comparative Biochemistry and Physiology Part A: Physiology, 111(Supplement 2): 183-189.

Mazzella L., Buia M. C., Gambi M. C., Lorenti M., Russo G. F., Scipione M. B., Zupo V., 1992, Plant-animal trophic relationships in the Posidonia oceanica ecosystem of the Mediterranean Sea: a review, [in:] PlantAnimal Interaction in the Marine Benthos, D. M. John, S. J. Hawkins, J. H. Price (eds), Systematics Association Special, Volume No. 46, Clarendon Press, Oxford: 165-187.

Mazzella L., Buia M. C., Gambi M. C., Lorenti M., Russo G. F., Scipione M. B., Zupo V., 1995, Organizzazione trofica nell'ecosistema a Posidonia, [in:] La Posidonia oceanica, F. Cinelli, E. Fresi, C. Lorenzi, A. Mucedola (eds), Roma: 31-47.

Meiner A., 2010, Integrated maritime policy for the European Union-consolidating coastal and marine information to support maritime spatial planning, Journal of Coastal Conservation, 14(Supplement 1): 1-11.

Milazzo M., Chemello R., Badalamenti F., Riggio S., 2000, Molluscan assemblages associated with photophilic algae in the Marine Reserve of Ustica Island (Lower Tyr- rhenian Sea, Italy), Italian Journal of Zoology, 67(Supplement 3): 287-295.

Newell P., Clavier J., 1997, Quantitative structure of soft substrate macrobenthos of Fiji's great astrolabe lagoon, Proceedings of the 8th International Coral Reef Symposium, 1: 455-458.

Orth R. J., Heck K. L., van Montfrans J., 1984, Faunal communities in seagrass beds: A review of the influence of plant structure and prey characteristics on predator-prey relationships, Estuaries, 7(Supplement 4): 339-350.

Paine R. T., 1969, A Note on Trophic Complexity and Community Stability, The American Naturalists, 103: 91-93.

Picone F., Buonocore E., D’Agostaro R., Donati S., Chemello R., Franzese P. P., 2017, Integrating natural capital assessment and marine spatial planning: A case study in the Mediterranean sea, Ecological Modelling, 361: 1-13.

Pitacco V., Orlando-Bonaca M., Mavrič B., Popović A., Lipej L., 2014, Mollusc fauna associated with the Cystoseira algal associations in the Gulf of Trieste (Northern Adriatic Sea), Mediterranean Marine Science, 15(Supplement 2): 225-238.

Purchon R. D., 1977, The Biology of the Mollusca, Second edition. Pergamon Press Ltd.

Rassweiler A., Costello C., Siegel D. A., 2012, Marine protected areas and the value of spatially optimized fishery management, Proceedings of the National Academy of Sciences, 109(Supplement 29): 11884-11889.

Richards G. W., 1983, Molluscan zonation on rocky shores in Malta, Journal of Conchology, 31: 207-224.

Root R. B., 1967, The niche exploitation pattern of the blue gray gnatcatcher, Ecological monographs, 37(Supplement 4): 317-350.

Rueda J. L., Gofas S., Urra J., Salas C., 2009, A highly diverse molluscan assemblage associated with eelgrass beds (Zostera marina L.) in the Alboran Sea: Micro-habitat preference, feeding guilds and biogeographical distribution, Scientia Marina, 73(Supplement 4): 679-700.

Russo G. F., 1989, La scelta dei descrittori morfo-funzionali nell'analisi dei sistemi bentonici: un approccio con la componente malacologica di una prateria a Posidonia oceanica, Oebalia, 15(Supplement 1): 213-228.

Russo G. F., Fresi E., Scardi M., 1985, Il popolamento a Molluschi dei fondi mobili del Golfo di Salerno: analisi strutturale in rapporto al trofismo, Oebalia, 11(Supplement 1): 339-348.

Russo G. F., Fresi E., Vinci D., Chessa L. A., 1984, Mollusk syntaxon of foliar stratum along a depth gradient in a Posidonia oceanica (L.) Delile meadow: Seasonal variability [in:] First international workshop on Posidonia oceanica beds, C. F. Boudouresque, A. Jeudy De Grissac, J. Olivier (eds), Marseille: 311-318. 
Russo G. F., Fresi E., Vinci D., Scardi M., 1986, Problemi e proposte sul campionamento della malacofauna di strato foliare nelle praterie di Posidonia oceanica (L.) Delile, Lavori SIM, 22: 15-28.

Russo G. F., Patti F. P., 2005, Early life history of two closely related gastropods, Rissoa auriscalpium and Rissoa italiensis (Caenogastropoda: Rissoidae), Marine biology, 147(Supplement 2): 429-437.

Salánki J., Farkas A., Kamardina T., Rózsa K. S., 2003, Molluscs in biological monitoring of water quality, Toxicology Letters, 140-141: 403-410.

Sánchez-Moyano J. E., Estacio F. J., García-Adiego E. M., García-Gómez J. C., 2000, The molluscan epifauna of the alga Halopteris scoparia in Southern Spain as a bioindicator of coastal environmental conditions, Journal of Molluscan Studies, 66(Supplement 4): 431-448.

Schoener T. W., 1974, Resource partitioning in ecological communities, Science, 185(Supplement 4145): 27-39.

Simboura N., Zenetos A., 2002, Benthic indicators to use in ecological quality classification of Mediterranean soft bottom marine ecosystem, including a new biotic index, Mediterranean Marine Science, 3(Supplement 2): 77-111.

Terlizzi A., Scuderi D., Fraschetti S., Guidetti P., Boero E., 2003, Molluscs on subtidal cliffs: patterns of spatial distribution, Journal of the Marine Biological Association of the United Kingdom, 83(Supplement 1): 165172.

Tews J., Brose U., Grimm V., Tielbörger K., Wichmann M. C., Schwager M., Jeltsch F., 2004, Animal species diversity driven by habitat heterogeneity/diversity: the importance of keystone structures, Journal of Biogeography, 31(Supplement 1): 79-92.

Urra J., Gofas S., Rueda J. L., Marina P., 2011, Molluscan assemblages in littoral soft bottoms of the Alboran Sea (Western Mediterranean Sea), Marine Biology Research, 7(Supplement 1): 27-42.

Vassallo P., Paoli C., Buonocore E., Franzese P. P., Russo G. F., Povero P., 2017, Assessing the value of natural capital in marine protected areas: A biophysical and trophodynamic environmental accounting model, Ecological Modelling, 355: 12-17.

WoRMS Editorial Board, 2018, World Register of Marine Species, Available from http://www.marinespecies.org at VLIZ, Accessed 2018-06-19. doi:10.14284/170.

Zuschin M., Hohenegger J., Steininger F., 2001, Molluscan assemblages on coral reefs and associated hard substrata in the northern Red Sea, Coral Reefs, 20(Supplement 2): 107-116. 